\title{
Recent results on B mixing and decay constants from HPQCD
}

\section{J. Shigemitsu ${ }^{* a}$, C. Davies ${ }^{b}$, E. Follana ${ }^{c}$, E. Gámiz ${ }^{d}$, E. Gregory ${ }^{b}$, P. Lepage Le $^{e}$ H. Na ${ }^{a}$ and $\mathbf{M}$. Wingate ${ }^{f}$}

${ }^{a}$ Department of Physics

The Ohio State University, Columbus, OH 43210, USA

${ }^{b}$ Department of Physics \& Astronomy

University of Glasgow, Glasgow G12 8QQ, UK

${ }^{c}$ Departamento de Física Teórica

Universidad de Zaragoza, E-50009 Zaragoza, Spain

${ }^{d}$ Department of Physics

University of Illinois, Urbana, IL 61801, USA

${ }^{e}$ Laboratory of Elementary Particle Physics

Cornell University, Ithaca, NY 14853, USA

${ }^{f}$ Department of Applied Mathematics and Theoretical Physics

University of Cambridge, Cambridge CB3 OWA, UK

E-mail: shige@mps.ohio-state.edu

We review recent results for $B_{d}$ and $B_{s}$ mixing parameters using MILC $N_{f}=2+1$ lattices, NRQCD b-quarks and AsqTad light quarks. Latest numbers for decay constants $f_{B}$ and $f_{B_{S}}$ are also presented. Combining our lattice results with experimental determinations of the mass differences $\Delta M_{d}$ and $\Delta M_{s}$ leads to an important ratio of elements of the CKM matrix, $\left|V_{t d}\right| /\left|V_{t s}\right|=0.214(1)(5)$ and an updated Standard Model number for the branching fraction $\operatorname{Br}\left(B_{s} \rightarrow \mu^{+} \mu^{-}\right)=3.19(19) \times 10^{-9}$. Preliminary new results for $f_{B_{s}}$ based on other actions are also described.

The XXVII International Symposium on Lattice Field Theory

July 26-31, 2009

Peking University, Beijing, China

\footnotetext{
${ }^{*}$ Speaker.
} 


\section{Introduction}

$B$ Physics, and in particular the study of $B$ and $B_{s}$ meson decays and mixing, remains an important part of Flavor Physics. Such studies enable consistency tests of the Standard Model and placing of bounds on New Physics effects. Lattice QCD is playing a crucial role in this effort by providing the necessary nonperturbative QCD inputs.

This talk describes recent results by the HPQCD collaboration on B meson mixing parameters, $f_{B_{s}} \sqrt{\hat{B}_{B_{s}}}, f_{B_{d}} \sqrt{\hat{B}_{B_{d}}}$ and their ratio $\xi$, the first fully consistent $N_{f}=2+1$ lattice QCD calculations of these quantities [1]. We work with four of the MILC coarse ( $a \approx 0.12 \mathrm{fm})$ lattices and two of the fine $(a \approx 0.09 \mathrm{fm})$ lattices. We use NRQCD for the $b$-quark and the AsqTad action for both valence and sea light quarks. Details are given in [1]. In the Standard Model the mass difference in the $B_{q}-\overline{B_{q}}(q=d, s)$ system is given by [2],

$$
\Delta M_{q}=\frac{G_{F}^{2} M_{W}^{2}}{6 \pi^{2}}\left|V_{t q} V_{t b}^{*}\right|^{2} \eta_{2}^{B} S_{0}\left(x_{t}\right) M_{B_{q}} f_{B_{q}}^{2} \hat{B}_{B_{q}} .
$$

In addition to well determined quantities, eq.(1.1) involves the combinations of CKM matrix elements $\left|V_{t q} V_{t b}^{*}\right|^{2}$ and the nonperturbative QCD factors $f_{B_{q}}^{2} \hat{B}_{B_{q}}$. The latter are determined from the matrix element of the four-quark operator ( $i, j$ are color indices that are summed over),

$$
O L \equiv\left[\bar{\Psi}_{b}^{i}(V-A) \Psi_{q}^{i}\right]\left[\bar{\Psi}_{b}^{j}(V-A) \Psi_{q}^{j}\right]
$$

sandwiched between the $B_{q}$ and $\bar{B}_{q}$ states.

$$
\langle O L\rangle^{\overline{M S}}(\mu) \equiv\left\langle\bar{B}_{q}|O L| B_{q}\right\rangle^{\overline{M S}}(\mu) \equiv \frac{8}{3} f_{B_{q}}^{2} B_{B_{q}}(\mu) M_{B_{q}}^{2} .
$$

One sees from eq.(1.1) that the ratio of the two CKM matrix elements $\left|V_{t d}\right|$ and $\left|V_{t s}\right|$ can be determined from

$$
\frac{\left|V_{t d}\right|}{\left|V_{t s}\right|}=\xi \sqrt{\frac{\Delta M_{d}}{\Delta M_{s}} \frac{M_{B_{s}}}{M_{B_{d}}}},
$$

once theory provides the important ratio

$$
\xi \equiv \frac{f_{B_{s}} \sqrt{B_{B_{s}}}}{f_{B_{d}} \sqrt{B_{B_{d}}}} .
$$

One of the main goals of [1] was to produce a state-of-the-art lattice calculation of $\xi$. We were able to determine this quantity with total error of $2.6 \%$.

\section{Hadronic Matrix Elements in the Effective Theory}

The $b$ quarks in our calculations are described by an effective theory NRQCD. Instead of the full QCD field $\bar{\Psi}_{b}$ of eq.(1.2) one works with fields $\bar{\Psi}_{Q}$ that create a heavy quark or $\bar{\Psi}_{\bar{Q}}$ that annihilate a heavy anti-quark. The $[V-A] \times[V-A]$ four-fermion operator becomes,

$$
O L^{e f f} \equiv\left[\bar{\Psi}_{Q}^{i}(V-A) \Psi_{q}^{i}\right]\left[\bar{\Psi}_{\bar{Q}}^{j}(V-A) \Psi_{q}^{j}\right]+\left[\bar{\Psi}_{\bar{Q}}^{i}(V-A) \Psi_{q}^{i}\right]\left[\bar{\Psi}_{Q}^{j}(V-A) \Psi_{q}^{j}\right] .
$$


In the effective theory one finds mixing with another operator at one-loop of $[S-P] \times[S-P]$ structure, even at lowest order in $1 / M$.

$$
O S^{e f f} \equiv\left[\bar{\Psi}_{Q}^{i}(S-P) \Psi_{q}^{i}\right]\left[\bar{\Psi}_{\bar{Q}}^{j}(S-P) \Psi_{q}^{j}\right]+\left[\bar{\Psi}_{\bar{Q}}^{i}(S-P) \Psi_{q}^{i}\right]\left[\bar{\Psi}_{Q}^{j}(S-P) \Psi_{q}^{j}\right] .
$$

Furthermore there is a tree-level dimension 7 correction to $O L^{\text {eff }}$ at $\mathscr{O}(1 / M)$.

$$
\begin{aligned}
O L j 1= & \frac{1}{2 M}\left[\left(\vec{\nabla} \bar{\Psi}_{Q} \cdot \vec{\gamma}(V-A) \Psi_{q}\right)\left(\bar{\Psi}_{\bar{Q}}(V-A) \Psi_{q}\right)\right. \\
& \left.+\left(\bar{\Psi}_{Q}(V-A) \Psi_{q}\right)\left(\vec{\nabla} \bar{\Psi}_{\bar{Q}} \cdot \vec{\gamma}(V-A) \Psi_{q}\right)\right]+\left[\bar{\Psi}_{\bar{Q}} \rightleftharpoons \bar{\Psi}_{Q}\right] .
\end{aligned}
$$

We have calculated the matching between matrix elements of the effective theory operators $O L^{e f f}$, $O S^{e f f}$ and $O L j 1$ and $\langle O L\rangle$ of eq.(1.2) in full QCD through $\mathscr{O}\left(\alpha_{s}, \Lambda_{Q C D} / M, \alpha_{s} /(a M)\right)$ and find

$$
\begin{aligned}
\langle O L\rangle^{\overline{M S}}(\mu)= & {\left[1+\alpha_{s} \rho_{11}\right]\left\langle O L^{\text {eff }}\right\rangle+\alpha_{s} \rho_{12}\left\langle O S^{\text {eff }}\right\rangle+} \\
& \langle O L j 1\rangle-\alpha_{s}\left[\zeta^{11}\left\langle O L^{\text {eff }}\right\rangle+\zeta^{12}\left\langle O S^{\text {eff }}\right\rangle\right]+\mathscr{O}\left(\alpha_{s}^{2}, \alpha_{s} \Lambda_{Q C D} / M\right) .
\end{aligned}
$$

The matching coefficients $\rho_{11}, \rho_{12}, \zeta^{11}$ and $\zeta^{12}$ are listed (for $\mu=M_{b}$ ) in [3].

The goal of lattice simulations is to obtain the matrix elements $\langle\hat{O}\rangle$, with $\hat{O}=O L^{\text {eff }}, O S^{\text {eff }}$ or $O L j 1$. To this end one calculates three-point correlators,

$$
C_{\alpha \beta}^{(4 f)}\left(t_{1}, t_{2}\right)=\sum_{\vec{x}_{1}, \vec{x}_{2}}\left\langle 0\left|\Phi_{\bar{B}_{q}}^{\alpha}\left(\vec{x}_{1}, t_{1}\right)\left[a^{6} \hat{O}(0)\right] \Phi_{B_{q}}^{\beta \dagger}\left(\vec{x}_{2},-t_{2}\right)\right| 0\right\rangle,
$$

together with two-point correlators

$$
C_{\alpha \beta}^{2 p t}(t) \equiv \sum_{\vec{x}_{1}, \vec{x}_{2}}\left\langle 0\left|\Phi_{B_{q}}^{\alpha}\left(\vec{x}_{1}, t\right) \Phi_{B_{q}}^{\beta \dagger}\left(\vec{x}_{2}, 0\right)\right| 0\right\rangle .
$$

One works with dimensionless operators $a^{6} \hat{O}$ which are kept fixed at the origin of the lattice. $\Phi_{B_{q}}^{\alpha}$ is an interpolating operator for the $B_{q}$ meson of smearing type " $\alpha$ ", and spatial sums over $\vec{x}_{1}$ and $\vec{x}_{2}$ ensure one is dealing with zero momentum $B_{q}$ and $\overline{B_{q}}$ incoming and outgoing states. Eq.(2.5) corresponds to a $B_{q}$ meson (or excitations thereof with the same quantum numbers) being created at time $-t_{2}$ which then propagates to time 0 where it mixes onto its anti particle a $\overline{B_{q}}$. The latter meson is annihilated at time $t_{1}$.

We have accumulated simulation data for a range in $t_{1}, t_{2}$ for three-point and in $t$ for two-point correlators, i.e. $1 \leq t_{1}, t_{2}, t \leq T_{\max }$ with $T_{\max }=24$ on the coarse lattices and $T_{\max }=32$ on the fine ensembles. As is well known, staggered quarks lead to meson two-point correlators that have both regular and time oscillating contributions. Hence a fit ansatz for eq.(2.6) would be

$$
C_{\alpha \beta}^{2 p t}(t) \equiv \sum_{j=0}^{N-1} b_{j}^{\alpha} b_{j}^{\beta} e^{-E_{j}(t-1)}+(-1)^{t} \sum_{k=0}^{\tilde{N}-1} \tilde{b}_{k}^{\alpha} \tilde{b}_{k}^{\beta} e^{-\tilde{E}_{k}(t-1)} .
$$

Similarly, the presence of oscillatory contributions makes fitting the three-point correlators, eq.(2.5) particularly challenging. The appropriate ansatz is

$$
\begin{aligned}
& C_{\alpha \beta}^{(4 f)}\left(t_{1}, t_{2}\right)=\sum_{j=0}^{N-1} \sum_{k=0}^{N-1} A_{j k}^{\alpha \beta} e^{-E_{j}\left(t_{1}-1\right)} e^{-E_{k}\left(t_{2}-1\right)}+\sum_{j=0}^{\tilde{N}-1} \sum_{k=0}^{N-1} B_{j k}^{\alpha \beta}(-1)^{t_{1}} e^{-\tilde{E}_{j}\left(t_{1}-1\right)} e^{-E_{k}\left(t_{2}-1\right)} \\
& \quad+\sum_{j=0}^{N-1} \sum_{k=0}^{\tilde{N}-1} C_{j k}^{\alpha \beta}(-1)^{t_{2}} e^{-E_{j}\left(t_{1}-1\right)} e^{-\tilde{E}_{k}\left(t_{2}-1\right)}+\sum_{j=0}^{\tilde{N}-1} \sum_{k=0}^{\tilde{N}-1} D_{j k}^{\alpha \beta}(-1)^{t_{1}}(-1)^{t_{2}} e^{-\tilde{E}_{j}\left(t_{1}-1\right)} e^{-\tilde{E}_{k}\left(t_{2}-1\right)} .
\end{aligned}
$$


In terms of the fit parameters in (2.7) and (2.8) the matrix elements we are interested in and which appear on the RHS of (2.4) are given by,

$$
\left\langle\overline{B_{q}}|\hat{O}| B_{q}\right\rangle=\frac{2 M_{B_{q}}}{a^{3}} \frac{A_{00}^{\alpha \beta}}{b_{0}^{\alpha} b_{0}^{\beta}} .
$$

In order to extract $\frac{A_{00}^{\alpha \beta}}{b_{0}^{\alpha} b_{0}^{\beta}}$, we have carried out simultaneous fits to $C_{\alpha \beta}^{(4 f)}\left(t_{1}, t_{2}\right)$ for $\alpha=\beta$ together with a matrix of two-point correlators $C_{\alpha \beta}^{2 p t}(t)$ with all possible combinations of smearings at source and sink. We used Bayesian fitting methods and typically employed $N=\tilde{N}=4 \sim 6$ number of exponentials.

\section{Chiral and Continuum Extrapolations}

After the fits to simulation data described above (see [1] for more details) one ends up with lattice results for $r_{1}^{3 / 2} f_{B_{q}} \sqrt{M_{B_{q}} \hat{B}_{B_{q}}}$ for each of the 6 MILC ensembles that we worked with. Here $r_{1}$ is a scale derived from the static potential which can be used to make dimensionful quantities dimensionless. We exploit the fact that the MILC collaboration has calculated $r_{1} / a$ for each of their ensembles. In order to make contact with the real world one needs to extrapolate both to the continuum (lattice spacing $a \rightarrow 0$ ) and to the chiral $\left(m_{\text {light }} \rightarrow m_{u / d}\right)$ limits. We do so by fitting to the following ansatz, inspired by chiral perturbation theory $(\mathrm{ChPT})$.

$$
r_{1}^{3 / 2} f_{B_{q}} \sqrt{M_{B_{q}} \hat{B}_{B_{q}}}=c_{1}\left[1+\frac{1}{2} \Delta f_{q}+c_{2}\left(2 m_{f}+m_{s}\right) r_{1}+c_{3} m_{q} r_{1}\right] \times\left[1+c_{4} \alpha_{s}\left(a / r_{1}\right)^{2}+c_{5}\left(a / r_{1}\right)^{4}\right] .
$$

$\Delta f_{q}$ includes the chiral logarithms including those specific to Staggered ChPT and was calculated by C.Bernard, J.Laiho and R.Van de Water [4]. We show chiral/continuum extrapolations for $r_{1}^{3 / 2} f_{B_{s}} \sqrt{M_{B_{s}} \hat{B}_{B_{s}}}$ and $r_{1}^{3 / 2} f_{B_{d}} \sqrt{M_{B_{d}} \hat{B}_{B_{d}}}$ in Fig.1 and for $\xi \sqrt{M_{B_{s}} / M_{B_{d}}}$ and for $\hat{B}_{B_{s}}$ in Fig.2. The red curves are the continuum extrapolated curves and the red circle gives the results at the physical point. Table I gives our error budget.

\section{Results}

Using central values coming from the physical (red) points in the figures and the errors summarized in Table I, we can now present our main results [1].

$$
\xi \equiv \frac{f_{B_{s}} \sqrt{B_{B_{s}}}}{f_{B_{d}} \sqrt{B_{B_{d}}}}=1.258(25)(21), \quad \hat{B}_{B_{s}}=1.33(5)(3)
$$

and using $r_{1}=0.321(5) \mathrm{fm}[5]$,

$$
f_{B_{s}} \sqrt{\hat{B}_{B_{s}}}=266(6)(17)\left(\frac{0.321}{r_{1}[f m]}\right)^{3 / 2} \mathrm{MeV}, \quad f_{B_{d}} \sqrt{\hat{B}_{B_{d}}}=216(9)(12)\left(\frac{0.321}{r_{1}[f m]}\right)^{3 / 2} \mathrm{MeV}
$$

where the first error comes from statistics + chiral extrapolation and the second is the sum of all other systematic errors added in quadrature. The result for $f_{B_{s}} \sqrt{\hat{B}_{B_{s}}}$ in eq.(4.2) is consistent with but more accurate than our previously published value of 281(21) MeV [6]. 

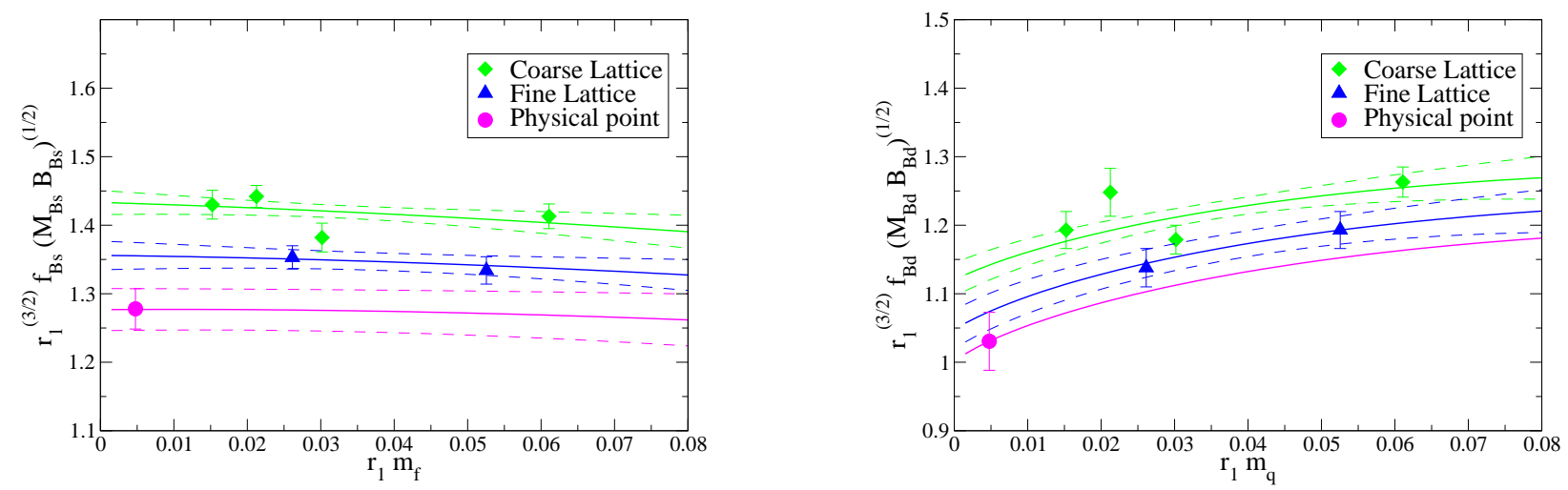

Figure 1: Chiral and continuum extrapolation of $r_{1}^{3 / 2} f_{B_{s}} \sqrt{M_{B_{s}} \hat{B}_{B_{s}}}, r_{1}^{3 / 2} f_{B_{d}} \sqrt{M_{B_{d}} \hat{B}_{B_{d}}}$. The red curve shows the continuum extrapolation and the "physical point is at $m_{\text {light }} / m_{s}=1 / 27.4$.

Combining our lattice result for $\xi$ with the experimentally measured mass differences $\Delta M_{d}=$ $0.507 \pm 0.005 \mathrm{ps}^{-1}[7]$ and $\Delta M_{s}=17.77 \pm 0.10 \pm 0.07 \mathrm{ps}^{-1}[8]$ leads to,

$$
\frac{\left|V_{t d}\right|}{\left|V_{t s}\right|}=0.214(1)(5)
$$

where the first error is experimental and the second from the lattice calculation presented here. Our Bag parameter result $\hat{B}_{B_{s}}$ can be combined with experimental $\Delta M_{s}$ and $\tau\left(B_{s}\right)$ to form the Standard Model prediction for the branching fraction $\operatorname{Br}\left(B_{s} \rightarrow \mu^{+} \mu^{-}\right)$[9]. We find

$$
\operatorname{Br}\left(B_{s} \rightarrow \mu^{+} \mu^{-}\right)=3.19(19) \times 10^{-9},
$$

improving on the previous accuracy available.

\begin{tabular}{|c|c|c|c|}
\hline source of error & $f_{B_{s}} \sqrt{\hat{B}_{B_{s}}}$ & $f_{B_{d}} \sqrt{\hat{B}_{B_{d}}}$ & $\xi$ \\
\hline \hline stat. + chiral extrap. & 2.3 & 4.1 & 2.0 \\
residual $a^{2}$ extrap. & 3.0 & 2.0 & 0.3 \\
uncertainty & & & \\
\hline$r_{1}^{3 / 2}$ uncertainty & 2.3 & 2.3 & - \\
$g_{B^{*} B \pi}$ uncertainty & 1.0 & 1.0 & 1.0 \\
$m_{s}$ and $m_{b}$ tuning & 1.5 & 1.0 & 1.0 \\
operator matching & 4.0 & 4.0 & 0.7 \\
relativistic corr. & 2.5 & 2.5 & 0.4 \\
\hline Total & 6.7 & 7.1 & 2.6 \\
\hline
\end{tabular}

Table 1: Errors in $\%$ for $f_{B_{s}} \sqrt{\hat{B}_{B_{s}}}, f_{B_{d}} \sqrt{\hat{B}_{B_{d}}}$ and $\xi$. 

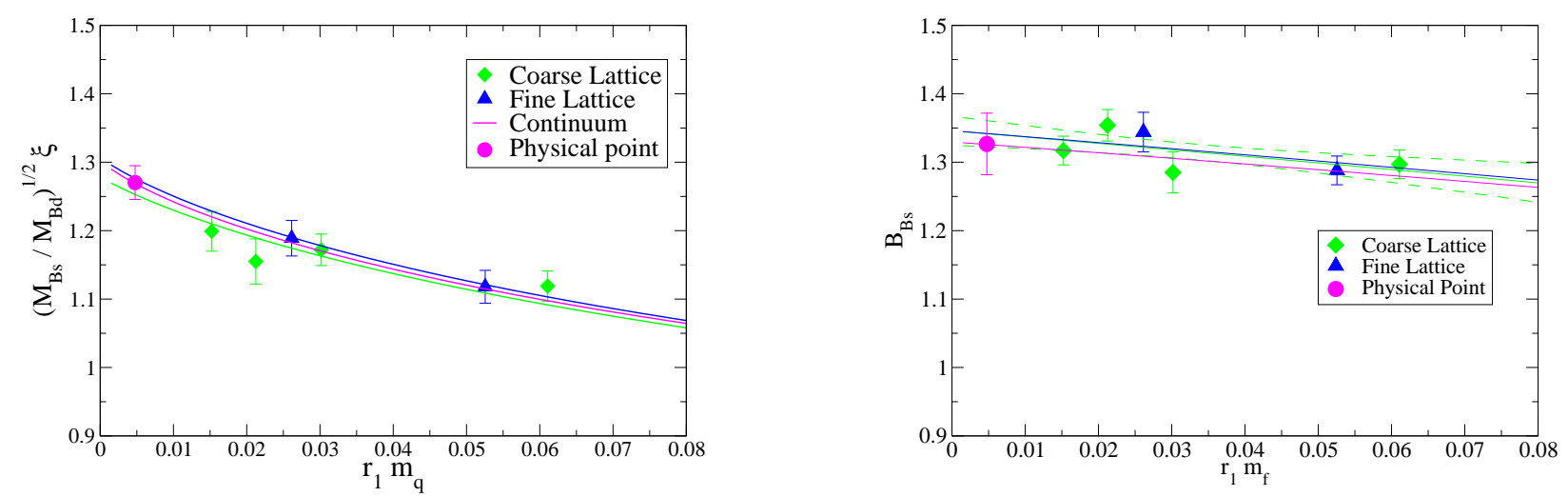

Figure 2: Same as Fig.1 for $\xi \sqrt{M_{B_{s}} / M_{B_{d}}}$ and for $\hat{B}_{B_{s}}$

Our fits to a matrix of $B_{q}$ two-point correlators has also allowed us to update previous results on $B_{d}$ and $B_{s}$ decay constants. The final numbers including all errors added in quadrature become,

$$
\begin{gathered}
\frac{f_{B_{s}}}{f_{B_{d}}}=1.226(26), \\
f_{B_{d}}=190(13)\left(\frac{0.321}{r_{1}[\mathrm{fm}]}\right)^{3 / 2} \mathrm{MeV}, \quad f_{B_{s}}=231(15)\left(\frac{0.321}{r_{1}[\mathrm{fm}]}\right)^{3 / 2} \mathrm{MeV} .
\end{gathered}
$$

These results for $f_{B_{q}}$ are consistent with but about one $\sigma$ lower than the values $f_{B_{d}}=216(22) \mathrm{MeV}$ and $f_{B_{s}}=260(29) \mathrm{MeV}$ given in $[10,11]$. The main difference between the analysis carried out here and in [10] is that in the latter case chiral extrapolations were done based only on coarse lattice data and furthermore no attempt was made to extrapolate explicitly to the continuum limit.

\section{5. $B_{s}$ Meson Decay Constant Using Other Actions}

The HPQCD collaboration has initiated a project of studying $B$ physics with NRQCD $b$-quarks and HISQ light quarks [12]. The hope is that by going from the AsqTad to the more highly improved HISQ action discretization errors will be further reduced, in particular those coming from taste breaking effects. We are also simulating the $B_{c}$ system with NRQCD $b$ - and HISQ charmquarks [13]. Here we present first preliminary results for the $B_{s}$ meson decay constant $f_{B_{s}}$ in Fig.3. One sees that the slope versus lattice spacing is significantly reduced as one moves to the HISQ action. Furthermore both actions lead to the same continuum limit within errors. We are pursuing other approaches to $B$ physics as well, in particular one based on relativistic HISQ $b$-quarks. Initial calculations using relativistic $b$-quarks show results for $f_{B_{s}}$ in good agreement with the NRQCDAsqTad and NRQCD-HISQ values presented here [14].

\section{Acknowledgments}

The numerical simulations were carried out on facilities of the USQCD Collaboration funded by the DOE, at the Ohio Supercomputer Center and at NERSC. 


\section{References}

[1] E.Gámiz et al. [HPQCD Collaboration]; Phys. Rev. D80, 014503 (2009).

[2] A.J.Buras, M.Jamin and P.Weisz; Nucl.Phys. B347, 491 (1990).

[3] E.Gámiz, J.Shigemitsu and H.Trottier; Phys. Rev. D77, 114505 (2008).

[4] We thank C.Bernard, J.Laiho and R.van de Water for communicating their results to us prior to publication.

[5] A.Gray et al. [HPQCD Collaboration]; Phys. Rev. D72, 094507 (2005).

[6] E.Dalgic et al. [HPQCD Collaboration]; Phys. Rev. D76, 011501(R) (2007).

[7] C.Amsler et al. Review of Particle Physics, Phys. Lett. B667:1, 2008.

[8] A.Abulencia et al. [CDF Collaboration]; Phys. Rev. Lett. 97, 242003 (2006).

[9] A.Buras; Phys.Lett. B 566, 115 (2003).

[10] A.Gray et al. [HPQCD Collaboration]; Phys. Rev. Lett. 95, 212001 (2005).

[11] M.Wingate et al. [HPQCD Collaboration]; Phys. Rev. Lett. 92, 162001 (2004).

[12] E.Gregory et al. [HPQCD Collaboration]; these proceedings.

[13] E.Gregory et al. [HPQCD Collaboration]; arXiv:0909.4462 [hep-lat].

[14] E.Follana et al. [HPQCD Collaboration]; these proceedings.

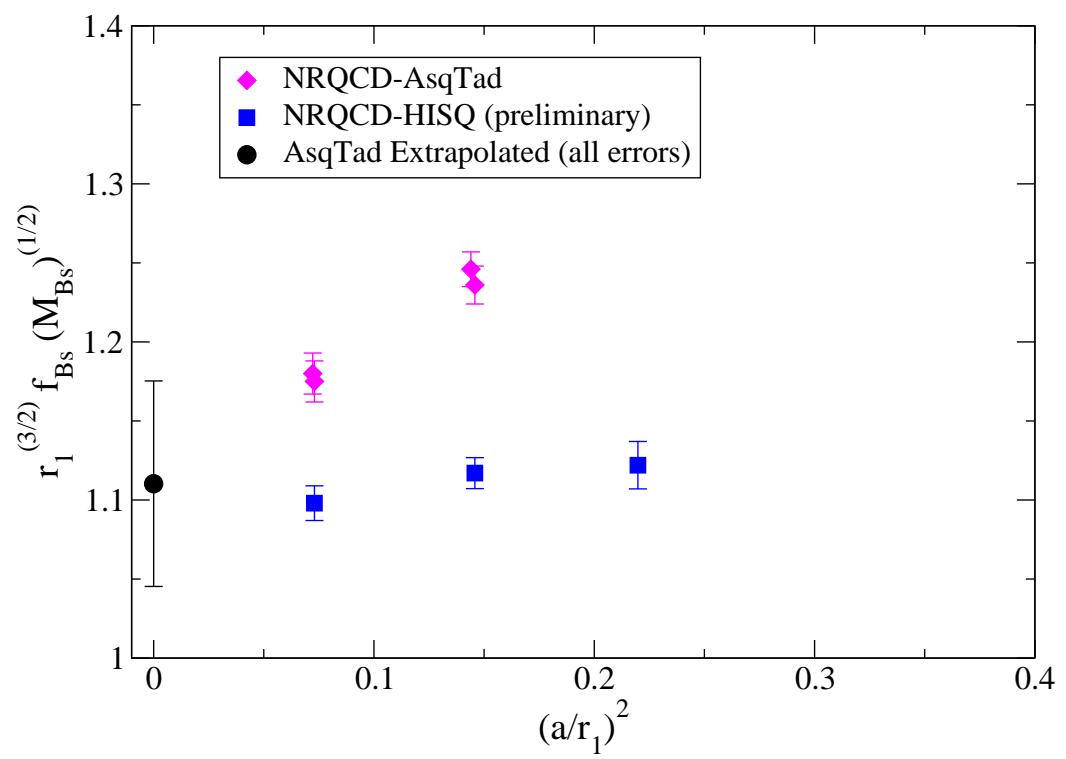

Figure 3: Comparison between NRQCD-AsqTad and NRQCD-HISQ calculations of the $B_{s}$ meson decay constant. 